\title{
Transfer of responsibility and accountability of patient care
}

\author{
Linda Deravin*1, Judith K. Anderson ${ }^{1,2}$ \\ ${ }^{1}$ Charles Sturt University, School of Nursing, Midwifery and Indigenous Health, Bathurst, NSW, Australia \\ ${ }^{2}$ University of Tasmania, Burnie, TAS, Australia
}

Received: December 8, 2020

Accepted: December 28, 2020

Online Published: 00-00-0000

\begin{abstract}
Objective: This paper reports on a study of clinical incidents related to the transfer of accountability and responsibility of patient care during clinical handover in three major health facilities in regional Australia. It aims to identify significant issues in the area of transferring accountability and responsibility.

Background: Although clinical handover is widely acknowledged as the process which transfers accountability and responsibility, issues occur particularly when this transfer is incomplete, shared or when one clinician feels an ongoing sense of responsibility for the patient.

Methods: A thematic analysis of incidents related to clinical handover was conducted on data collected at three regional settings within Australia in order to identify issues which had occurred during this process. The Incident Information Management System (IIMS) is a database that collects information about clinical incidents and near misses and relies on health staff to report them. The initial information retrieved from IIMs identified 3716 possible events for inclusion. A thematic analysis was undertaken of the data which identified transfer of responsibility and accountability as a key theme.

Results: The data related to the transfer of responsibility and accountability came to prominence in the incident reports in three ways. These included; identifying omissions, issues with information exchange and refusal to accept responsibility of care.

Conclusions: This study demonstrates the need for a more systematic approach regarding communication between health professionals regarding the transferability and accountability of patient care.

Relevance to clinical practice: Clinical handover remains a contentious issue regarding patient care and safety. Health professionals may benefit from this review of incidents related to clinical handover and consider some of the recommendations to improve clinical practice.
\end{abstract}

Key Words: Clinical handover, Responsibility and accountability, Communication, Health professionals

\section{INTRODUCTION}

Clinical handover is the process which transfers accountability and responsibility from one clinician to another which can be either verbal or written. ${ }^{[1]}$ Clinical handover is included in a health employee's work responsibilities involving tasks assigned by employers through position descriptions which an employee is held accountable to perform. ${ }^{[2]}$

Clinical handover is undertaken multiple times in a variety of places and situations between all levels of health care professionals. In a previous review of literature conducted by Anderson et al., ${ }^{[1]}$ the process of accountability and the transfer of responsibility of care was identified as a major

\footnotetext{
* Correspondence: Linda Deravin; Email: lderavin@csu.edu.au; Address: Charles Sturt University, School of Nursing, Midwifery and Indigenous Health, Panorama Ave, Bathurst, NSW, Australia.
} 
issue in delivering patient care safely. The purpose of this study was to further examine this issue by looking at a range of clinical incidents related to clinical handover to determine if this issue can be validated from practice. In this study only incidents where an event has occurred were included in the study.

\section{BACKGROUND}

Although clinical handover is widely acknowledged as the process which transfers accountability and responsibility, issues may occur particularly when this transfer is incomplete, shared or when one clinician feels an ongoing sense of responsibility for the patient. ${ }^{[3]}$ The relationship to policies is seen to be central, however responsibility and accountability is seen to be an aspect which requires more definition and development. $^{[4]}$

McMurray, Chaboyer ${ }^{[5]}$ identify the need for accountability within the process of clinical handover for accuracy and appropriateness of content and communication. Thomas, Schultz ${ }^{[6]}$ support the notion that accurate communication is an essential element of accountability. Berger, Sten ${ }^{[7]}$ recommend a dual responsibility. The clinician conveying the handover needs to be accurate, ensuring that all information is provided. However the clinician receiving the handover needs to accept personal responsibility for the patient and their care requirements and should seek additional information once the handover process is completed if needed.

Bost, Crilly ${ }^{[8]}$ indicate that responsibility exists for a good quality handover and that this may even be formalised with a comment such as "the patient is now yours". Between some disciplines e.g. the transfer from paramedic to emergency staff, the transfer of responsibility is seen to be complete when the move has occurred from the stretcher to the bed, ${ }^{[8]}$ the end of handover, the end of a shift or actual commencement of work. ${ }^{[1,3]}$ Even though there is much in the literature that supports how clinical handover could be improved, issues with the transfer of accountability and responsibility of patient care remain.

\section{METHODS}

This study involved a review of all clinical incidents at three large regional hospitals in rural Australia. The clinical incidents reviewed in this study were extracted from the Incident Information Management System (IIMS), an electronic database which records all incidents and events related to patient care and the delivery of health services. All events that occurred in the acute care setting within a 12 month period, were included. Ethical approval for this study was provided by the relevant Human Research and Ethics Committee. All data was de identified to protect patient and staff identities. To enable data tracking, sites were labelled A, B and C.

The total combined incidents retrieved from the IIMs during the time period of the study identified 3716 events. Two culls of the information were performed by the researchers. The first cull removed all incidents that were not related to clinical handover. This left a combined total of 519 from sites $\mathrm{A}, \mathrm{B}$ and $\mathrm{C}$. The second cull identified reoccurring themes and each event was allocated to one or more themes. Each researcher initially identified relevant themes and discussions took place until concordance was reached. This resulted in a total of 269 incidents for inclusion in this study. Those incidents that were unable to be linked to a theme were considered to be isolated incidents and were removed from the study (see Table 1).

\section{RESULTS}

The theme of the transfer of responsibility and accountability came to prominence in the incident reports in three ways. These included: identifying omissions, issues with information exchange and refusal to accept responsibility of care.

Table 1. Culling process

\begin{tabular}{ll}
\hline Data/Cull & Events remaining \\
\hline Initial 12-month data collected & 3,716 \\
$1^{\text {st }}$ cull removing all incidents not related to clinical handover & 519 \\
$2^{\text {nd }}$ cull removing isolated incidents which were unable to be linked to a reoccurring theme & 269 \\
\hline
\end{tabular}

\subsection{Identifying omissions}

Many incident reports which were considered in this study had a focus on assigning accountability. In health care there are many things which are not the sole responsibility of a single person. When this is the case, there can be doubt about whose "fault" it is when something is not done or when an omission in care occurs. These incidents which were related to accountability included events related to consent such as this one where the clinical handover from one staff member to another discovered that consent had not been obtained: 
"MrX was bought to the operating theatre at approximately 2230. I attempted to check his details with him, but he was unable to answer me appropriately. It was discovered that the consent form was blank. Dr Y was notified. He filled out the consent form and attempted to speak to the patient but the patient was unable to consent. This was not witnessed by any nursing staff. The patient required the surgery so it proceeded without a consent form. Pt. described as disorientated preoperatively. No consent obtained prior to surgery. Surgery proceeded due to the urgent requirement for this procedure. No next of kin or guardian available or contactable (Site $C)$."

In this scenario the patient was described as being disorientated pre-operatively and in urgent need of surgery. However, the assignment of responsibility is clearly being diverted away from the staff member who completed the report. We see this again in the following quote:

"Unclear verbal and written communication in relation to $C$ (cervical) spine clearance. Patient sitting upright and looking around on arrival to ward. Still complaining of neck pain. Nil ortho notes post review. Verbal handover given to in charge of ward unclear and insufficient (Site B)."

This demonstrates an important aspect of clinical handover which is the benefit of another person's perspective. In this case the identification of an omission that the first staff member may not have been aware of. This identification of omissions is also seen in the following reports from two different facilities:

"Arrived on ward with no IVC (intravenous cannula), no regular medications written up and no IVF written up as is NBM from midnight. Also no pain relief written up (Site A)."

"No written or verbal communication regarding patient's infectious status, from Nurse escort or documents from ward. Nil flag in iPM (inpatient management) (Site B).”

"Missed observation of patient and PCA (patient controlled anaesthesia) overnight, potentially increased staff failure to recognise deterioration and escalation of care, earlier than detected (0715). No SAGO (Standard Adult General Observation chart) observation recorded from 2130, no PCA observation recorded overnight (Site B)."

Although this, like the previous report is written in a manner which is designed to protect the writer from negative consequences, it can also be seen that from a greater systems focus on quality improvement and reporting, the time when clinical handover occurs can be used as a checking mechanism. It is a time when errors and omissions can be identified allowing intervention to occur.

Published by Sciedu Press
"Patient admitted with? stroke to the XXX ward. Patient was admitted via ED (emergency department). Patient arrived on the ward at 0430 on the 23.12.14 and no referral to speech pathology was attended. Patient referred from case conference and the speech assessment was attended at 1102 (Site C).”

Handing over the responsibility and accountability of care between shifts is an opportunity to prevent the omission of care and treatments that should be provided. When communication transfer completely breaks down staff are left wondering how the following situation could have been prevented. In this event staff are allocated to patients yet the staff member has not acknowledged that the patient who is allocated to them is part of their responsibility.

"Patient was not looked after by nursing staff for a whole evening shift. Patient returned to ward after a debridement of sacral pressure injury at 13.40 hours. Morning staff took a set of obs then. When night staff arrived, there was no handover available. The staff that was allocated the patient on the hand-over sheet stated she was not aware that she was to be looking after the patient. The shift in-charge was not aware that the patient was not being looked after that shift (Site C)."

"Patients wound care attended to back of head/neck. Dressing had not been attended according to documentation for at least 10 days resulting in degradation of wound and wound dressing melting and adhering to patient's skin and hair. Patient required precision removal of glue/melted dressing using scalpel and multiple shaving cream applications to aid in dissolving of adhesive (Site A)."

From the incidents reviewed, the accounting or shifting of blame for omission of care was evident. However clinical handover obviously lends itself to identifying and rectifying omissions of care which can improve patient care. Fostering a culture of open communication without allocating blame would improve patient outcomes.

\subsection{Information exchange}

However issues were identified that related to a transfer of responsibility and accountability including poor communication and information exchange. Similarly to the previous theme, the incident reports related to this theme demonstrate a desire on the part of the writer to protect themselves. The incident described below is an example of this:

"Pt (patient) alerted NUM (nursing unit manager) that she had not received her 6am Parkinson medication, stating she was given 3 tablets, 2 of which she identified as Panadol but the 3rd she is adamant was not her Sinemet. Pt normally self-medicates at home. RN from N/D (night duty) contacted 
at home and states she administered the right medications to the patient and felt the patient was slightly confused this morning at 6am. This was not verbalised at handover and the patient did not inform morning staff of her concern until after N/D RN had left the unit (Site A)."

"Received patient at 18.30 hrs from [tertiary referral hospital], no proper documentation received from [tertiary referral hospital], no copies of medication chart, obs chart, fluid balance chart ,ECG etc, just received a doctors transfer letter and nurses transfer letter. Patient had insertion of pacemaker..., also has a history of stroke (Site B).”

As a strategy to improve the communication between staff information systems such as PowerChart, eMR and information tools such as ISBAR have been adopted in the clinical setting. However it is evident from the following quote that these are not used to their maximum efficiency and are reliant on the human factor to enter and retrieve information required for the successful transfer of accountability and responsibility of care.

"Pt with reported/recorded allergy in eMR (electronic medical record)/Powerchart (powerchart is an electronic medical record) not placed on allergy diet in Powerchart. Pt was ordered a diet without any allergy restrictions. Near miss. Pt could have been given allergens thus resulting in allergic reaction. Issue with transfer of care as staff entering diet into Powerchart did not enter allergies into diet order. An allergies report is done in the diet office to capture Pt where Powerchart has sent an allergy alert to CBORD (Site C)."

"Patient had not had a set of vital observations completed since morning staff check at 1,230 hrs. Was handed over that patient was between the flags. Attended by night staff manually at 2,355 hrs, patient between the flags (Between the flag-common reference to a patient safety improvement program which highlights where standard observation go outside normal parameters allowing the clinician to recognise the deteriorating patient ) (Site B)."

Sharing information can be influenced by familiarity with other staff. When handing over care to staff, and in environments which are familiar, information exchange is enhanced. However when information needs to be exchanged to staff who operate external to the familiar environment, issues often ensue due to these relationships being more remote or disconnected and that they may possibly never interact with the information receiver again.

"Patient accepted by vascular team for transfer to [major tertiary referral centre] over weekend, bed manager informed - nil beds at time of request and advised will transfer over weekend. Patient sent via air ambulance regardless of no bed available in [major tertiary referral centre] (Site C).”

"Handed over on transfer pt clinical condition had deteriorated and? questionable pedal pulses present and required intervention by ?Vascular/Medical team... No documentation of deterioration of patient noted in progress notes by either nursing staff or rehabilitation team (Site A)."

In the event of a life threatening or emergency situation responsibility and accountability are more important but due to their stressful nature errors in communication are more likely to occur when people are under pressure to perform their duties yet have limited time to do so.

"Pt was brought into theatre for a Category 1 C-Section (Caesarean section). Pt was on the table and awake, staff and anaesthetist were waiting for surgeon to arrive. Surgeon rushed into theatre and gowned up, instructed for the scrub nurse for a scalpel and did not consult with anaesthetics if they were ready or had administered anaesthetics. Surgeon made an incision into lower abdomen whist pt was still awake. Pt started screaming and distressed. Surgeon stopped as soon as he noticed pt was not asleep. Pt was anaesthetised and procedure continued as normal once pt was asleep (Site $C$ ).”

“Rapid response was call for pt's BP of 180/80. Reluctance shown from Obstetric team regarding $R R$ (rapid response), sending RR team away before they visualised pt, stating they had it covered and were not requiring assistance. JMO (junior medical officer) from RR team over hearing nursing conversation regarding legal requirements, became argumentative towards nursing staff, stating there is NO legal requirement to call a $R R$ if observations in 'red zone', with nursing staff needing to use own 'clinical judgement', also stating there is not policy supporting the between the flags protocol. Obstetric consultant and registrars abrupt and dismissive towards nursing staff involved (Site B)."

Factors that influence effective information exchange during clinical handover included the desire of staff to protect themselves from negative consequences, and to apportion blame to systems rather than those who input the data into systems. Lack of familiarity between health professionals and stressful situations (particularly life threatening or emergency episodes of care) can have a direct influence on the amount or quality of information that is shared.

\subsection{Refusal to accept responsibility of care}

The line of responsibility of care can become unclear when there is a crossover of different departments and professions. When this occurs it may lead to the patient not receiving care in a timely manner. 
"Police refusing to escort a schedule 1 patient to a declared mental health facility. Patient brought in by police on a section 22 with homocidal ideation and psychosis. Schedule 1 written and police escort requested on schedule. Second schedule 1 written after first schedule not valid after 24 hours, this also requested police escort. Ed Doctors, CL (clinicians) mental health and psychiatrist all agreed this patient a risk to others. Police aware of this. Inspector XXXX deemed this patient to be low risk and refused to provide an escort. XXX Ambulance attended ED $x 4$ to transport. Eventually, patient cannulated, chemically sedated, physically restrained and escorted by (Emergency department doctor in an ambulance) to mental health facility after more than 25 hours in ED (Site A)."

In the following incidents we see situations where staff refuse to provide handover which is part of their professional responsibility. In cases where patients are being transferred to other wards for ongoing care and treatment.

"Patient scheduled for admission to ward from Dialysis unit. Patient was bought to ward by wardsman, no handover was provided to nursing staff either in person or via phone. Nursing staff were not made aware of patient's arrival on the ward. Ward staff phoned through to dialysis to obtain a phone handover on patient. Was advised that dialysis staff were too busy at this time to provide handover to ward (Site B)."

"Patient booked on the Emergency list. Theatre wardsman sent to collect the patient from the ward. On arrival to OT (operating theatre) the Anaesthetic nurse asked the Theatre wardsman where the ward nurse was so that she could be provided with a handover of the patient's condition and care. The wardsman noted that there was no ward nurse escorting the patient to Theatre. No hand over of patient care to the Operating Theatre Staff (Site B)."

Not all incidents demonstrated a lack of clarity. Some demonstrated clear refusal to accept responsibility of care when delegated to do so. In some cases the IIMS system allows the staff to protect themselves by describing the situation and their reasoning for refusing this delegation of care.

"Patient was brought down to ICU (Intensive Care Unit) from ED without any notification. staff acuity was over, two staff members were missing from the ward (transferring patients out to accept the ICU patient from ED) there was another ICU patient requiring one nurse and the other nurse to look after the $x 4$ HDU (2 of which are BIPAP [Bi Level positive airway pressure] dependant) and $x 1 C C U$ (Coronary Care Unit) therefore no one was able to admit and accept care of the ICU patient that was transferred down to ICU from ED without any notification. the ED patient had to be transferred

Published by Sciedu Press back to ED as it was unsafe. when ED was phoned about the incident the in charge nurse said that she received a phone call from ICU staff stating to 'bring it down' when no staff did such thing as it was unsafe to take care of a critical patient without appropriate staffing. when phoned the ADON (Assistant Director of Nursing) the ADON stated that she said to bring the patient down despite the ADON not being in attendance or knowing what was happening in the intensive care unit at that specific time, she was under the assumption that all the ward patients were already cleared out (Site B)."

In other situations of refusal to accept delegation of care and treatment the incident reporting system again functions to protect the reporter from legal issues which may ensue when instructions are not followed:

"Delay in management of Pt care. Syntocinon not commenced by midwife for many hours against repeated oral request and Drs written orders (Site A)."

Refusal of care can be related to a lack of clarity about professional boundaries and responsibilities but was also seen in the refusal to provide a clinical handover or outright refusal of to follow reasonable medical orders for the ongoing care and treatment of a patient.

\section{Discussion}

\subsection{Different expectations about information needs}

With the significant effort that has been placed in developing easy to use clinical handover mnemonics within a range of settings this brings us to consider the expectation of various staff in obtaining the relevant information that they need. ${ }^{[9]}$ Different health professionals require different information in order to undertake their work effectively. ${ }^{[10]}$ This may influence the focus of what is or is not communicated between individuals, which can impact on the effectiveness of clinical handover and potentially result in the omission of important information for the next health professional who has the responsibility of care for the patient.

During clinical handover it is important that both parties recognise the needs of the other and ensure that information that is exchanged is sufficient. This will require a range of health professionals understanding and accepting that all have a role in providing patient care and that responsibility for the transfer of information is shared. ${ }^{[11,12]}$ This means that the information provider needs to give a comprehensive clinical handover and that the information receiver also has the responsibility to request clarification and additional information if required.

This study showed that regardless of the mnemonics being used or where the handover takes place that communication 
between individuals remains challenging. The opportunity for the identification of omissions is ideally placed within the handover process yet needs to take place in a collegial and professionally respectful manner. Conversely when there is no established collegial relationship between information providers and receivers, this study also highlighted that omissions in information were more likely to occur. ${ }^{[1]}$

Health care environments can be stressful. This needs to be taken in to consideration when providing handover as when people are stressed, errors and omissions in communication are more likely to occur. ${ }^{[13]}$ As demonstrated in the theme of information exchange errors continue to occur more frequently in stressful environments.

\subsection{Transference of blame and avoidance of ownership}

As mentioned previously, clinical handover is an ideal time to identify omissions in care and to ensure that measures are put in place that support good patient outcomes. However when staff do not have good working relationships this often results in blaming others when errors or omissions occur. Hewitt, Chreim \& Forster ${ }^{[14]}$ identify that several different frames (states of mind) are used when staff in health care environments are completing incident forms. One of these frames was the fear of being blamed and this frame was supported through the evidence in this study. It is easier to deflect the ownership of an error on to another person rather than accepting the responsibility for that error. People may feel vulnerable that their clinical practice or professional integrity may come in to question. As demonstrated in this study incident reports were often couched in such a way as to deflect blame from the reporter of the incident and allocating blame to another clinician for poor care or patient outcomes.

Incident information management systems are designed to collect information about incidents and near misses. ${ }^{[15]}$ From this review it was evident that some staff used the incident information system to vent their frustration or anger about clinical care rather than taking the approach of identifying systems errors which require intervention to prevent an incident from recurring.

\subsection{Education}

Education regarding clinical handover should include legal aspects and professional accountability. During the clinical handover process, opportunities to engage in learning that support less experienced staff can occur. In this study the identification of omissions and ability to rectify gaps in care demonstrated an opportunity to support less experienced staff to learn more about current practices. To support this learning education on clinical handover practices should be implemented in both undergraduate learning ${ }^{[16]}$ and as professional development for current practitioners.

The value of simulation to support this learning process has been demonstrated in the literature. ${ }^{[16-18]}$ Simulation allows novice clinicians to gain confidence in their ability to participate and deliver handover of patient care. Education for undergraduate clinicians should include a variety of tools to support clinical handover and expose them to a wide range of clinical scenarios which may require different types of information to be delivered. ${ }^{[1]}$ This research identifies the role of issues and omissions as occurring reasonably frequently. Although this research demonstrates a tendency of people "reporting" issues to shift the blame onto those in the previous shift, verbal handovers in particular are a "joint" venture with the receiving clinician also having responsibility to question any situation which lacks clarity or seems incomplete. ${ }^{[19]}$ This is an easy and responsive way to overcome communication issues, improving safety and accountability. Students should be placed in situations where they face these types of issues and omissions and simulate possible responses and ways to challenge such issues. ${ }^{[20]}$

\subsection{Relevance to clinical practice}

Clinical handover remains a contentious issue regarding patient care and safety. Health professionals may need to be further involved in review of incidents related to clinical handover and consider interventions from a cultural and systemic perspective rather than a personal one. ${ }^{[4]}$ When considering these deficits from a personal perspective, staff become defensive and shift blame, whereas identifying them from a cultural perspective of supporting other staff to work better would assist in producing better outcomes for patients. Similarly considering them from a systemic perspective would allow the identification of tools that support better handover practices $^{[21,22]}$ and education that would also improve clinical practice.

\subsection{Limitations}

Limitations acknowledged within this study is that the information was sourced from a database reliant on user input therefore the accuracy and lack of bias could not be determined when users input information into the system. The IIMS system captures information in instances where there is an event or potential mishap and has the potential to negatively skew perceptions about the transfer of information related to handover. This study was conducted as a snapshot view of clinical incidents and does not account for any changes or developments in improving clinical handover since the data was extracted. 


\section{Conclusions}

The evidence provided within this study supports that issues with communication of clinical handover remain and can lead to poor patient outcomes. The transfer and accountability of patient care is a dual responsibility of both the receiver and the provider of clinical handover. Clinicians are frequently worried about professional consequences regarding negligence when omissions in care occur. What is important is the need to recognise that although clinical handover tools support communication this is more likely to be threatened when dealing with health professionals who are unfamiliar or remote to the usual workplace, or in stressful situations.

\section{CONFlicts of InTEREST Disclosure}

The authors declare they have no conflicts of interest.

\section{REFERENCES}

[1] Anderson J, Malone L, Shanahan K, et al. Nursing bedside clinical handover - an integrated review of issues and tools. Journal of Clinical Nursing. 2015; 24(5-6): 662-71.

[2] Iqbal N, Rees M, Backer C. Decision making, responsibility and accountability in community mental health teams. Mental Health Practice. 2014; 17(7): 26-8. https://doi.org/10.7748/mhp201 4.04.17.7.26.e926

[3] Chin GSM, Warren N, Kornman L, et al. Transferring responsibility and accountability in maternity care: clinicians defining their boundaries of practice in relation to clinical handover. BMJ Open. 2012; 2(5): 1-9. PMid: 22952159. https://doi.org/10.1136/bmjope $\mathrm{n}-2011-000734$

[4] Spurgeon P, Sujan MA, Cross S, et al. Safety and Culture: Theory and Concept. In: Spurgeon P, Sujan MA, Cross S, Flanagan H, editors. Building Safer Healthcare Systems: A Proactive, Risk Based Approach to Improving Patient Safety. Cham: Springer International Publishing; 2019. 45-50 p. https://doi.org/10.1007/978-3 -030-18244-1_4

[5] McMurray A, Chaboyer W, Wallis M, et al. Implementing bedside handover: strategies for change management. Journal of Clinical Nursing. 2010; 19(17-18): 2580-9. PMid: 20522156. https: //doi.org/10.1111/j.1365-2702.2009.03033.x

[6] Thomas MJW, J. Schultz T, Hannaford N, et al. Failures in transition: Learning from incidents relating to clinical handover in acute care. Journal for Healthcare Quality. 2012; 35(3): 49-56. PMid: 22268639. https://doi.org/10.1111/j.1945-1474.2011.00189.x

[7] Berger JT, Sten MB, Stockwell DC. Patient handoffs: Delivering content efficiently and effectively is not enough. International Journal of Risk \& Safety in Medicine. 2012; 24(4): 201-5. PMid: 23135334. https://doi.org/10.3233/JRS-2012-0573

[8] Bost N, Crilly J, Wallis M, et al. Clinical handover of patients arriving by ambulance to the emergency department - A literature review. International Emergency Nursing. 2010; 18(4): 210-20. PMid: 20869662. https://doi.org/10.1016/j.ienj. 2009.11.006

[9] Spinks J, Chaboyer W, Bucknall T, et al. Patient and nurse preferences for nurse handover-using preferences to inform policy: a discrete choice experiment protocol. BMJ Open. 2015; 5(11): e008941. PMid: 26560060. https://doi.org/10.1136/bmjopen-2015-00894 1

[10] Johnson M, Sanchez P, Zheng C. The impact of an integrated nursing handover system on nurses' satisfaction and work practices. Journal of Clinical Nursing. 2016; 25(1-2): 257-68. PMid: 26769213. https://doi.org/10.1111/jocn. 13080
[11] Coleman K, Redley B, Wood B, et al. Interprofessional interactions influence nurses' adoption of handover improvement. ACORN: The Journal of Perioperative Nursing in Australia. 2015; 28(1): 10 https://doi.org/10.26550/jpn.281.01

[12] Wood K, Crouch R, Rowland E, et al. Clinical handovers between prehospital and hospital staff: literature review. Emergency Medicine Journal. 2015; 32(7): 577-81. PMid: 25178977. https : //doi.org/10.1136/emermed-2013-203165

[13] Zakrison TL, Rosenbloom B, McFarlan A, et al. Lost information during the handover of critically injured trauma patients: a mixedmethods study. BMJ Quality \& Safety. 2015: bmjqs-2014-003903.

[14] Hewitt T, Chreim S, Forster A. Sociocultural factors influencing incident reporting among physicians and nurses: understanding frames underlying self-and peer-reporting practices. J Patient Saf. 2014; 1-9.

[15] Anderson JE, Kodate N, Walters R, et al. Can incident reporting improve safety? Healthcare practitioners' views of the effectiveness of incident reporting. International Journal for Quality in Health Care. 2013; 25(2): 141-50. PMid: 23335058. https: //doi.org/10.1093/intqhc/mzs081

[16] Malone L, Anderson J, Manning J. Student participation in clinical handover-an integrative review. Journal of Clinical Nursing. 2016; 25(5-6): 575-82.

[17] Collins G. Using simulation to develop handover skills. Nursing times. 2014; 110(8): 12.

[18] Brown R, Rasmussen R, Baldwin I, et al. Design and implementation of a virtual world training simulation of ICU first hour handover processes. Australian Critical Care. 2012; 25(3): 178-87. PMid: 22436543. https://doi.org/10.1016/j. aucc. 2012.02.005

[19] Tobiano G, Ting C, Ryan C, et al. Front-line nurses' perceptions of intra-hospital handover. Journal of Clinical Nursing. 2020; 29(13-14): 2231-8. PMid: 32043671. https://doi.org/10.1111/jocn.1 5214

[20] Malone L, Anderson J, Manning J. Student participation in clinical handover-an integrative review. Journal of Clinical Nursing. 2016; 25(5-6): 575-82.

[21] Merten H, van Galen LS, Wagner C. Safe handover. BMJ. 2017; 359 : j4328-j. PMid: 28993308. https://doi.org/10.1136/bmj.j4 328

[22] Pucher PHMDPM, Johnston MJMBBM, Aggarwal RMMAPF, et al. Effectiveness of interventions to improve patient handover in surgery: A systematic review. Surgery. 2015; 158(1): 85-95. PMid: 25999255. https://doi.org/10.1016/j.surg.2015.02.017 